\title{
Ambient cosmology and spacetime singularities
}

\author{
Ignatios Antoniadis ${ }^{1,2, a}$, Spiros Cotsakis ${ }^{3,4, b}$ \\ ${ }_{1}^{1}$ Albert Einstein Center for Fundamental Physics, Institute for Theoretical Physics, Bern University, Sidlerstrasse 5, 3012 Bern, Switzerland \\ ${ }^{2}$ Ecole Polytechnique, 91128 Palaiseau, France \\ ${ }^{3}$ Department of Physics, CERN, Theory Division, 1211 Geneva 23, Switzerland \\ ${ }^{4}$ School of Applied Mathematics and Physical Sciences, National Technical University, 15780 Athens, Greece
}

Received: 17 September 2014 / Accepted: 11 January 2015 / Published online: 28 January 2015

(C) The Author(s) 2015. This article is published with open access at Springerlink.com

\begin{abstract}
We present a new approach to the issues of spacetime singularities and cosmic censorship in general relativity. This is based on the idea that standard 4-dimensional spacetime is the conformal infinity of an ambient metric for the 5-dimensional Einstein equations with fluid sources. We then find that the existence of spacetime singularities in four dimensions is constrained by asymptotic properties of the ambient 5-metric, while the non-degeneracy of the latter crucially depends on cosmic censorship holding on the boundary.
\end{abstract}

\section{Introduction}

The geometry and physics of braneworlds [1-4] and holographic ideas [5] such as the AdS/CFT correspondence [6,7] have provided conclusive evidence that new structures may exist in higher dimensions described by some metric $g_{5}$, with the 4-dimensional, general relativistic world confined in a suitable subspace $g_{4}$. The precise nature of the theory describing our universe that forms the basis of this is likely a version of string theory whose realization is presently unknown, and the same is true in such frameworks of the possible resolution of the well-known issues of Einstein's theory, such as the existence and nature of spacetime singularities and the justification of cosmic censorship.

Concerning the problem of singularities [8,9] and its possible resolution in higher dimensions, we note that the issue is further complicated by the appearance of new singularities in the geometry of the extra dimensions and the associated problems that this brings about, cf. [10-17] (and

Spiros Cotsakis: On leave from the University of the Aegean, 83200 Samos, Greece.

\footnotetext{
a e-mail: ignatios.antoniadis@cpht.polytechnique.fr

be-mail: skot@aegean.gr
}

references therein). Also, cosmic censorship [18] seems to emerge as an inherent property of 4-dimensional general relativistic metrics $g_{4}$, unconnected to the higher-dimensional structures presumably responsible to the relativistic singularity resolution.

In this work, we view the 4-dimensional relativistic world as a suitable asymptotic and holographic limit of structures that may exist only in higher dimensions. We know [19] how to asymptotically split a 5 -dimensional space $g_{5}$ containing a 'braneworld' [20-23] $g_{4}$, and starting with our asymptotic splitting solutions in the form $\left(M \times \mathbb{R}, g_{5}=a^{2}(y) g_{4}+d y^{2}\right)$, in the first part of this paper we introduce a new way to connect the properties of the 4-dimensional general relativistic world to a possible higher-dimensional theory (in 5 dimensions).

The basic idea of this work is that our world is the conformal infinity of a certain 'ambient' metric in one higher dimension. (We use the adjective 'ambient' to declare an analogy with the Fefferman-Graham ambient construction [24].) The ambient metric ( $\left.V=M \times \mathbb{R}, g_{+}\right)$satisfies the 5-dimensional Einstein equations with fluid sources, but it is constructed locally in an open neighborhood of the boundary, 4-dimensional conformal geometry $\left(M,\left[g_{4}\right]\right)$, and has a suitable metric $\left.\stackrel{g}{g}\right|_{M}$ in the conformal class as its conformal infinity. Because of its dependence on formal power series, this construction relies on both asymptotic as well as holographic properties of the higher-dimensional space, and possesses various novel properties. However, our approach is distinct from the general braneworld approach as well as the AdS/CFT and related approaches, in that in the former the brane cannot be the conformal infinity of the bulk geometry without spoiling the solutions completely, whereas in the latter one does not deal with gravitational effects on the boundary.

This paper falls into three parts. The first part, up to Sect. 3 , deals with the general motivation and outlines the basic features of our approach. In the next section we discusssome 
of the elements of standard braneworld cosmology relevant to the present context, and in Sect. 3 we outline in a series of distinct steps an alternative approach and method we use to deal with several of the features of the standard braneworld model. In Part 2, which includes Sects. 4-6, we expand on the various justifications of the steps involved in our construction and describe the normal form of the ambient metric, its conformal infinity and the asymptotic conditions, which are all key elements of ambient cosmology. In the third part of this work, Sects. 7 and 8, we discuss implications of this construction for the existence of spacetime singularities and the question of cosmic censorship in the 4-dimensional boundary of the ambient world.

\section{The standard braneworld cosmology}

In previous works [20-23], we studied the asymptotic properties of bulk 5-geometries $\left(V, g_{5}\right)$ containing an embedded 4-dimensional braneworld $\left(M, g_{4}\right)$ that was either a 4-dimensional Minkowski, or de Sitter, or Anti-de Sitter spacetime, and showed that, in general, asymptotic solutions have a form dictated by the method of asymptotic splittings [19], namely,

$a(y)=y^{p} \sum_{i=0}^{\infty} c_{i} y^{i / s}, \quad y \rightarrow 0$,

where the first constant $c_{0}$ in the series is nonzero (this is the dominant balance). Here, $y$ denotes the coordinate of the extra dimension in the 5-dimensional geometry $g_{5}$, where

$g_{5}=a^{2}(y) g_{4}+d y^{2}$,

$g_{4}$ being a 4-dimensional braneworld metric with signature $(-+++)$, and $a(y)$ is a warp factor in the $g_{5}$ geometry. The metric $g_{5}$ is taken to satisfy the 5-dimensional Einstein equations

$G_{A B}=T_{A B}$,

where $A, B=1,2,3,4,5$, and $T_{A B}$ is the stress tensor of an analog of a perfect fluid with equation of state $P=\gamma \rho$, where the 'pressure' $P(y)$ and the 'energy density' $\rho(y)$ depend only on $y,{ }^{1}$ filling the 5-dimensional geometry (other cases like a bulk scalar field or a mixture of bulk fluids considered in [20-23] also lead to the general form (2.1) for the warp factor of the 5-dimensional geometry (2.2)). These equations become, for the metric (2.2), a dynamical system of the form $\dot{x}=f(x)$, with the solution vector having the form $x=(a, \dot{a}, \rho)$, and $f$ being a suitable, smooth field

\footnotetext{
${ }^{1}$ In the following, we drop the quotes for notational simplicity, even if $T_{A B}$ is not a perfect fluid in the usual cosmological sense.
}

(cf. the references mentioned above). The asymptotic solutions for the energy density $\rho$ also have a form similar to (2.1).

Further, the constants $c_{i}, i=0,1,2, \ldots$ in (2.1) are determined recursively by the method of asymptotic splittings starting at 0th order with the dominant balance form $c_{0} y^{p}, p=m / l \in \mathbb{Q}, m \in \mathbb{Z}, l \in \mathbb{N}$ (with the dominant balance constant $c_{0}$ nonzero), and proceeding in a term-by-term fashion, while $s$ is defined to be any common multiple of the denominators of the positive eigenvalues of the Kovalevskaya matrix (cf. [19]).

This procedure leads to asymptotic solutions given generically by (2.1) (and similarly for the other unknowns), that is, Puiseux or Fuchsian series (meaning series with fractional exponents, and with or without a constant first term, respectively) describing the geometry locally in a small neighborhood around the location of the brane at $y=0$, or at infinity.

The braneworld setup described above has the following important properties: In the presence of a non-trivial fluid

- all 5-dimensional solutions are singular at a finite, arbitrary distance from the position of the brane, and the metric $g_{5}$ cannot be continued to arbitrary values in the $y$-dimension;

- the properties of the metric $g_{4}$ do not follow from those of the bulk metric $g_{5}$;

- there is no conformal infinity for the 5-dimensional geometry.

These properties are common not only for the models in [20-23] but, in fact, they are characteristic for all models of references [10-17] (and related references therein). The first point means that there are genuine singularities in the metric and the curvatures in the 5-dimensional geometry which do not allow the metric to be continued smoothly beyond them.

For the second point, we note that in most models of this sort the 4-metric is either taken to be fixed (e.g., Minkowski), or satisfying a 4-dimensional version of the Einstein equations with induced matter fields on the braneworld. In the latter case, although there are important differences, singularities are a typical feature of the evolution, in much the same way as in standard general relativity.

For the third point in the enumeration above, we note that in braneworld models the brane represents some kind of 'boundary' for the 5-dimensional spacetime, often a domain wall with suitable boundary conditions, but it can never be a conformal boundary. The reason is very simple: Trying to bring a solution of the form (2.2) for the warp factor of the 5-dimensional geometry (having all the required properties analyzed in detail in the references given above) to a suitable 'conformal infinity' form $\tilde{g}_{5}=\Omega^{2} g_{5}$ by multiplying it by a conformal factor, would completely destroy the properties of the original solution. A consequence of this is that there 
is no possibility of a holographic interpretation and no way to realize a boundary CFT.

A closely related issue is that an AdS/CFT or related approach (such as the so-called 'holographic renormalization cf. e.g., [25]) could not really be used for the problems we have in mind here, simply because there is no gravity on the boundary. In such approaches, one starts from solutions in the bulk satisfying Einstein's equations with sources there, and then one produces a conformal structure on the boundary on which a CFT resides without any mention of possible gravitational effects on the boundary.

\section{The ambient cosmology}

In this paper, we follow an inverse route and present a new approach that is distinctly different from any AdS/CFT-based or braneworld approach. Starting from a given metric defined on the boundary, we then consider the conformal structure of that boundary metric in the sense of [26], and use that to construct in a series of steps, a 5-dimensional metric with the property that after the construction it returns a suitable 4-metric belonging to the conformal structure on the boundary that we started with. In this sense, our approach is closer in spirit to the original Fefferman-Graham construction.

In the resulting 'ambient', 4-dimensional universe introduced here, the classical singularities and the question of cosmic censorship in the 4-dimensional conformal boundary of the 5-dimensional ambient space acquire new meanings. The general procedure consists of starting with a conformal structure on a 4-dimensional boundary, and constructing the ambient 5-metric corresponding to that structure which satisfies the 5-dimensional Einstein-fluid equations and has a nice conformal infinity.

In particular, we prove that corresponding to each 4metric $g$ in the conformal class $\left[g_{4}\right]$ of another, well-behaved, 4-metric $g_{4}$ on the 4-boundary $M$, that is, $g_{4}=\Omega^{2} g$, there is a new 4-metric $\left.\stackrel{\circ}{g}\right|_{M}$, a constant rescaling of $g_{4}$, which is the conformal infinity of the ambient 5-metric $g_{+}$of the metric $g$. The ambient metric $g_{+}$is defined on the 'ambient' spacetime $V=M \times \mathbb{R}$ and satisfies the 5-dimensional Einstein equations with a fluid source.

More specifically, we show how to extend the 5-metric (2.2) corresponding to the 'nice' 4-metric $g_{4}$, to a new, 5-dimensional metric $g_{4,+}$ (this is the ambient metric) on $V$ [a point in $V$ has coordinates $\left(x^{\mu}, w\right)$ ], such that the latter has a nice conformal infinity. Then we show that any 4-metric $g$ on the boundary $M$ that belongs to the conformal class of $g_{4}$ has itself an ambient 5-metric $g_{+}$with conformal infinity $\left(M,\left.\stackrel{\circ}{g}\right|_{M}\right)$ which is a constant rescaling of the nice metric $g_{4}$. Hence, our construction produces from a given metric $g \in\left[g_{4}\right]$ its ambient metric whose conformal infinity $\left.\stackrel{\circ}{g}\right|_{M}$ acquires various important and improved properties over the original 4-metric $g$.

The aforementioned extension is achieved in a series of steps as follows:

1. Take a 4-dimensional, non-degenerate 'initial' metric $g_{\text {IN }}\left(x^{\mu}\right)$ on spacetime $M$. This step essentially involves the Penrose conformal method [26].

2. Conformally deform $g_{\text {IN }}$ to a new metric $g_{4}=\Omega^{2} g_{\text {IN }}$ by choosing a suitable conformal factor $\Omega$. This step connects the 'bad' metric $g_{\text {IN }}$ with the 'nice', non-degenerate, and non-singular metric $g_{4}\left(x^{\mu}\right)$.

3. Using the method of asymptotic splittings for the 5dimensional Einstein equations with an arbitrary (with respect to the fluid parameter $\gamma$ ) fluid (2.3), solve for the 5-dimensional metric $g_{5}=a^{2}(y) g_{4}+d y^{2}$ and the matter density $\rho_{5}$.

4. Transform the solutions of step 3 to suitable factored forms of the general type, (divergent part) $\times$ (smooth part).

5. Construct the 'ambient' metric in normal form, $g_{+}$, for the 5-dim Einstein equations (2.3) with a suitable fluid.

6. $\left(M,\left[g_{4}\right]\right)$ is the conformal infinity of $\left(V, g_{+}\right)$, that is, $\mathscr{I}=\partial V=M$.

7. The metric $g_{+}$is conformally compact. This means that a suitable metric $\stackrel{\circ}{g}$ constructed from $g_{+}$extends smoothly to $V$, and its restriction to $M,\left.\stackrel{\circ}{g}\right|_{M}$, is non-degenerate (i.e., maintains the same signature also on $M$, cf. [24] for this definition).

8. The conformal infinity $M$ of the ambient metric $g_{+}$of any metric in the conformal class $\left[g_{4}\right]$ is controlled by the behavior of a constant rescaling of the 'nice' metric $g_{4}$.

9. As a conformal manifold, $\left(M,\left[g_{4}\right]\right)$ has no singularities. This means that there is always a regular metric on $M$ : the metric $\stackrel{\circ}{g} \mid M$ belonging to $\left[g_{4}\right]$ is regular. (See Sect. 7.)

10. Cosmic censorship on $\left(M,\left[g_{4}\right]\right)$ is equivalent to the validity of the asymptotic condition satisfied by the ambient metric $\left.\stackrel{\circ}{g}\right|_{M}$. (See Sect. 8.)

Some explanatory remarks are in order here. The initial 4-metric $g_{\text {IN }}$ of step 1 is any metric with a conformal infinity. For example, de Sitter, Anti-de Sitter, or Minkowski metrics, when written is suitable forms using the conformal method [26], show explicitly their conformal infinities. Similarly, for the Schwarszchild, Reissner-Nordstrom, or Kerr metrics, $g_{\text {IN }}$ represents their maximal analytic extensions (e.g., the Kruskal, double-null forms, etc.). The metric $g_{\text {IN }}$ written in this way, has a regular region which (like in the Einstein static universe for the exact models) is bounded by certain hypersurfaces, the conformal boundary. This boundary represents the various infinities of the metric, but most importantly also contains the singularities (in the sense of the classical 
singularity theorems [27]), previously located at some finite point not at infinity.

For example, in the FRW spaces, the bounding hypersurfaces of the metric $g_{\mathrm{IN}}$ represent points at infinity, but also contain the various singular points as some finite part of the conformal boundary at infinity. So for instance the singularity at $t=0$ is represented by a spacelike surface (generally part of the bounding hypersurfaces) at infinity. This is the meaning of the conformal method [26], and it applies similarly to other exact spacetimes (see also [27]). This is very convenient for what follows because a $t=0$, proper time singularity surface, which would prevent us from comparing the situation with a better metric in connection with the ambient construction, has now moved to infinity, and we can proceed with our 5-dimensional constructions even for singular 4-metrics (see below and especially Sect. 7).

In step 2, the term 'nice' has a suitable technical meaning giving better asymptotic properties over the metric $g_{\mathrm{IN}}$ that we started with. In this sense, considering the conformal form of spacetimes in step 1 and having the classical singularities at the conformal boundary by that construction, has the advantage that we may basically kill off the infinities and singularities of the $g_{\mathrm{IN}}$ metric simply by multiplying by a suitable conformal factor, and so end up with a regular spacetime, $g_{4}$, for instance, Minkowski or de Sitter or Antide Sitter. In all cases, we will have a boundary $M$ possessing a conformal structure $\left[g_{4}\right]$, where any two metrics belonging to $\left[g_{4}\right]$ are conformal transformations of each other. For instance, taking $g_{\mathrm{IN}}$ to be the flat FRW metric in its conformal form, or an exact black hole spacetime (like the maximally extended spacetimes mentioned above), we may choose a suitable, smooth conformal factor in step 2, and obtain the Minkowski metric for $g_{4}$.

We also note that in steps 1-2, we do not impose any dynamical equations for the metric $g_{\mathrm{IN}}\left(x^{\mu}\right)$ or $g_{4}$. In this way, we avoid the usual problems of having to decide which of the two, conformally related metrics represents a 'physical' metric among the two conformal 'frames' (cf. [28-30] and references therein). It is an important feature of the ambient construction that in the end, for any two conformally related, 4-dimensional metrics belonging to the conformal structure [ $\left.g_{4}\right]$ of $M$, their ambient metrics restricted on the boundary $M$ can be chosen to be not very different from the restricted ambient metric corresponding to $g_{4}$.

In step 3, the $g_{4}$ metric is the one from step 2. It may also be $g_{\text {IN }}\left(x^{\mu}\right)$ from step 1 , provided we exclude the points with coordinates $x^{\mu}$ on the singular boundary where the metric becomes infinite. One may wonder whether we are allowed to exclude such 'ideal' points from the metric $g_{\mathrm{IN}}\left(x^{\mu}\right)$ in the sought-for 5-dimensional ansatz $a^{2}(y) g_{\mathrm{IN}}\left(x^{\mu}\right)+d y^{2}$, because such 5 -dimensional solutions are obviously not defined there. However, since we do not impose any field equations on $M$, we do not know the domain of definition of the metric $g_{\mathrm{IN}}\left(x^{\mu}\right)$, even locally, starting from initial data on $M$. Therefore the only way to proceed is if we 'move' all possible singular finite points of the metric $g_{\mathrm{IN}}\left(x^{\mu}\right)$ to become ideal points at infinity, so that $g_{\mathrm{IN}}\left(x^{\mu}\right)$ is regular elsewhere on $M$. This is the purpose of using the conformal method on $g_{\mathrm{IN}}\left(x^{\mu}\right)$. Then, in step 3, we build asymptotic 5-dimensional solutions in the form of formal series expansions using the technique of asymptotic splittings [19]. This method produces solutions for the warp factor and the density of the form $a(y)=y^{p_{1}} \sum_{i=0}^{\infty} c_{i} y^{i / s}, \quad \rho(y)=y^{p_{2}} \sum_{i=0}^{\infty} d_{i} y^{i / s}, \quad y \rightarrow$ 0 . All such solutions are singular in a finite distance $y_{s}$ from the boundary located at $y=0$ in the variable $y-y_{s}$. For notational simplicity we set $y_{s}=0$ here and in everything below, but this has the apparent effect of moving the singularity from the point at finite distance $y_{s}$ from the boundary, where it was originally located, to the boundary of the ambient space itself at $y=0$. We also note that according to the method of asymptotic splittings, the denominator $s$ appearing in such expansions is the same for all components of the solution $x(y)=(a, \dot{a}, \rho)$ of the dynamical system $\dot{x}=f(x)$ representing the 5-dimensional Einstein equations with the aforementioned fluid source.

Steps 4, 5 are studied in Sect. 4. In step 4, we show how to convert solutions of step 3 to the factored forms, $a(r)=r^{\kappa_{1}} \xi(r), \rho(r)=r^{\kappa_{2}} \zeta(r)$ as $r \rightarrow 0$, which contain a divergent, power-law part, and a smooth, convergent part. It is curious that this step necessarily introduces an asymmetry in the $y$-dimension, in the sense that we no longer can have invariance under the symmetry $y \rightarrow-y$ in the extra dimension $y$. The transformation that prepares the solutions to be brought to a suitable form that implies the convergence we need (so that we may apply various relevant theorems, see the first half of Sect. 4), is a very simple change in the parametrization of the extra dimension, $r=r(y)$, which itself has certain properties: (1) It is consistent with the (later) property that the 5-dimensional spacetime has a conformal boundary at $y=0,(2)$ it is well-defined only in the halfspace $y>0,{ }^{2}(3)$ the boundary in the new parameter $r$ is again located at 0 , and (4) it strongly highlights the particular features of the series solutions we use.

We continue, in the second half of Sect. 4, with step 5: the ambient metric has the form $g_{+}=w^{-n}\left(\sigma^{2}(w) g_{4}\left(x^{\mu}\right)\right.$ $\left.+d w^{2}\right), n \in \mathbb{Q}^{+}$, as $w \rightarrow 0$, with $\sigma(w)$ a smooth (infinitely differentiable) function such that $\sigma(0)$ is a nonzero constant. The metric $g_{+}$(essentially $g_{5}$ up to constant rescalings in different variables) solves the 5-dimensional Einstein equations (2.3) with a suitable fluid with density $\rho_{+}(w)$ that has the generic form $\rho_{+}=w^{-z} \theta(w), z \in \mathbb{Z}$ and $\theta(w)$ smooth. Both functions $w(r)$ and $w(y)$ are well-defined and invertible.

\footnotetext{
2 This is similar to the situation with Minkowski space being the boundary of the AdS space in the AdS/CFT correspondence.
} 
Step 6 is studied in Sect. 5 after we have introduced the metric $\stackrel{\circ}{g}$. For this step, we prove that when $\stackrel{g}{g}$ is restricted on $M$, it becomes a metric in the conformal class $\left[g_{4}\right]$. In the first part of Sect. 5, we also complete the construction of the ambient metric started in the previous section, for two special cases which need for their treatment the introduction of the metric $\stackrel{\circ}{g}$.

The property of conformal compactness of step 7 is shown later in Sect. 5. It means that we can always pass to the metric $\stackrel{g}{g}=\omega(w) g_{+}, \omega$ smooth (in a slight abuse of language, we will also call $g$ the ambient metric), which extends smoothly on $V=M \times \mathbb{R}$, and when restricted to the first factor $M$ gives the metric $\stackrel{\circ}{g} \mid M$ that is non-degenerate. Although $\stackrel{\circ}{g}$ does not solve the Einstein equations like $g_{+}$, they both share the same conformal boundary and so everything we say about the behavior of $\stackrel{\circ}{g}$ on $M$ holds essentially also for $g_{+}$.

For step 8 we show in Sect. 6 that for a metric $g \in\left[g_{4}\right]$, the difference of the two ambient metrics $\stackrel{\circ}{g}-\stackrel{\circ}{g}_{4}$ has a restriction on $M$ which satisfies a set of asymptotic conditions. These conditions imply that the ambient metric $\left.\stackrel{g}{g}\right|_{M}(0)$, for any $g \in\left[g_{4}\right]$, is a constant rescaling of $g_{4}$, and therefore has an improved behavior over the original 4-metric $g$.

We thus end up with the following situation. There is an ambient manifold $\left(V, g_{+}\right)$on which we have the Einstein equations (2.3) valid for the ambient metric $g_{+}$as defined above. The ambient spacetime has a boundary where only a conformal structure $\left[g_{4}\right]$, not a unique metric, is defined. The construction is local in nature and shows that the asymptotic structure of the ambient 5-dimensional geometry $g_{+}$at its conformal boundary $\left(M,\left[g_{4}\right]\right)$ has conformal infinity the metric $\left.\stackrel{g}{g}\right|_{M}$ as defined above. For any given 4-metric $g \in\left[g_{4}\right]$, its ambient 5-metric is given by

$g_{+}=w^{-n}\left(g_{w}+d w^{2}\right)$,

where

$g_{w}=\sum_{i=0}^{\infty} c_{i} w^{i} g, \quad w \rightarrow 0$,

with the coefficient of $g$ being a convergent formal power series of $w$, such that $g_{0}=c_{0} g\left(c_{0}=\sigma(0)\right.$ is a nonzero constant). Hence, $g_{w}$ represents a 1-parameter family of boundary metrics constructed recursively by the method of asymptotic splittings, and it is asymptotic to $\sigma^{2}(w) g$, where $\sigma^{2}$ is the asymptotic smooth sum of the power series. This sum, in the limit $w \rightarrow 0$, gives the metric $\left.\stackrel{g}{g}\right|_{M}(0)$ belonging in the conformal structure of the boundary $\left[g_{4}\right]$ and having constant conformal factor.

This construction has implications for singularities and the question of cosmic censorship on the boundary 4-spacetime $M$ as we discuss in Sects. 7 and 8.

\section{The normal form of the ambient cosmology}

It is not immediately obvious that a general Fuchsian formal series leads to a well-defined function; in fact, such a series generally will not because it either contains general powers of the form $\sum_{i=-\infty}^{\infty} c_{i} y^{\eta_{i}}, \eta_{i} \in \mathbb{Q}$, or log terms, or both, cf. [31].

However, in this section we show that due to its special form, that is, having equal denominators (all being equal to $s$ ), the series factor in Eq. (2.1) converges provided we introduce an asymmetry in the $y$ dimension, and this gives an important first step to the realization of our basic construction in this and the following sections.

To begin, we introduce the parameter

$r=y^{1 / s l}, \quad p=m / l, \quad m \in \mathbb{Z}, \quad l \in \mathbb{N}$,

into (2.1) and notice that this is real provided we choose $y>0$, and well-defined since $s, l$ are positive integers by definition. This implies that we can have no symmetry of the form $y \rightarrow-y$ in $V$. Then it follows that (4.1) is an invertible transformation because $\mathrm{d} r / \mathrm{d} y=(1 / s l) y^{1 / s l-1}>0$.

Since $r \rightarrow 0$ when $y \rightarrow 0$, the Puiseux series (2.1) then trivially becomes a formal power series in disguise,

$a(r)=r^{s m} \sum_{i=0}^{\infty} c_{i} r^{i l}, \quad r \rightarrow 0$.

In this new form the convergence of the series in (4.2) follows because according to a theorem of Borel (cf. Ref. [32], p. 300), when the coefficients $c_{i}$ are real, the formal power series $\sum_{i=0}^{\infty} c_{i} r^{i}$ always converges to a smooth function $\sigma: I=(-\epsilon, \epsilon) \rightarrow \mathbb{R}$, for some $\epsilon>0$. A basic point in the proof of Borel's theorem is to use the mean value theorem of differential calculus and the constants $c_{i}$ to extend the derivative $\sigma^{(n)}$ as a continuous function everywhere in $I$ by assigning it, at 0 , the value $\sigma^{(n)}(0)=c_{n}, n=0,1,2, \cdots$. The resulting function $\sigma$ will also be real analytic in a small neighborhood around each nonzero point of the interval $I$. Therefore the warp factor will assume the form ${ }^{3}$

$a(r)=r^{\kappa} \sigma(r), \quad$ as $\quad r \rightarrow 0, \quad \kappa=s m \in \mathbb{Z}$,

so that $a$ will always be the product of some smooth (in fact real analytic) function times the factor $r^{\kappa}$. We note that because of the $y$-asymmetry we must necessarily have that $r>0$.

Now since $p$ is a parameter in the theory (determined by the structure of the field equations and the method of asymptotic splittings), there is the issue of understanding

\footnotetext{
3 Actually, the theorem of Borel is a special case of Ritt's theorem about the convergence of formal power series with complex coefficients, cf [32], p. 299. Such a power series will always be asymptotic to an analytic function $\sigma$ on an angular-shaped sector $\mathcal{S}$, that is we have $\sigma(z) \sim \sum_{i=0}^{\infty} c_{i} z^{i}, z \rightarrow 0$, on $\mathcal{S}$.
} 
the physical significance of the solutions as $p$ varies and, in particular, in the limit when $p \rightarrow \infty$ in (2.1). We showed above that when $p$ is bounded, the warp factor reduces to the canonical form (4.3), and so the 5-dimensional geometry (2.2) becomes

$g_{5}(r)=r^{2 s m}\left(\sigma^{2} g_{4}+r^{-2 q} d r^{2}\right), \quad q=s m-s l+1$.

We note here that $q \neq 1$, iff $m \neq l$ (i.e., $p \neq 1$ ). In this form, $\sigma$ is a new formal power series with coefficients determined recursively by the method of asymptotic splittings. We use again the same letter $\sigma(r)$, but now $r$ stands for the rescaled variable $r / s l$ which is also necessarily positive, and there is an overall additional constant factor $(1 / s l)^{2 s m}$ multiplying the brackets (again not shown for simplicity). To get $\sigma$, one considers the dominant part $f^{(0)}$ of the vector field, and the eigenvalues of the Kowalevskaya matrix $K=D f^{(0)}(A)-\operatorname{diag} P$ (where the dominant balance is $A t^{P}$ ) which are rational numbers, and where $s$ is defined to be the least common multiple of their denominators.

When the exponent $q$ defined in Eq. (4.4) is different from one we may introduce a new parameter $w$ (not necessarily positive this time), with

$d w=r^{-q} d r$.

Because $y>0$, we see that $d w / d r=y^{-q / s l}$ and $d w / d y=$ $(1 / s l) y^{-p}$ are positive, and so both reparametrizations are invertible. Then the 5-dimensional geometry (4.4) becomes

$g_{+}=w^{-n}\left(\sigma^{2}(w) g_{4}\left(x^{\mu}\right)+d w^{2}\right), \quad w \rightarrow 0$,

where

$-n=\frac{2 m}{l-m}=\frac{2 p}{1-p}$.

We call $g_{+}$, which stands for $g_{5}(w)$ (possibly rescaled by a constant factor of the form $(1-q)^{-2 s m /(1-q)}$ if necessary), the normal form of the ambient metric. The exponent $n$ satisfies the following properties:

- $1-p \neq 0$

- $-n<0$;

- $n=n(p)$, and in fact, $-n \neq-2$;

- $-n \rightarrow-2$, as $p \rightarrow \infty$.

Before we discuss the properties of this enumeration, we note here that there are two cases, for $p=1$ (already excluded from the discussion above when we assumed which $q \neq 1$ ) and when $p \in(0,1)$ (when $w \rightarrow 0$ ), that are not included in the above discussion. For these two cases, the prefactor in the normal form of the ambient metric $g_{+}$in Eq. (4.6) tends to zero and is not divergent. We shall discuss these two cases separately in the next section, when we introduce the metric $\stackrel{\circ}{g}$.
Returning to the enumeration above, the first property means that the exponent in the ambient metric (4.6) is always well-defined. We note that $\sigma$ is a new smooth (in fact, real analytic) function, for which we keep the same symbol as before. The second property justifies the name 'normal form' which we gave to the ambient metric (4.6), in analogy to the Fefferman-Graham (FM) ambient metric construction [24]. Their brilliant work provides an existence theorem for the ambient metric in the case of the 5-dimensional Einstein equations with a cosmological constant, and they also show that their ambient metric $g_{+}=w^{-2}\left(\sigma^{2} g_{4}+d w^{2}\right)$ is essentially unique. However, the third property of $n$ implies that our ambient metric is distinct from the FM metrics [the latter always have the constant characteristic exponent equal to -2 in place of our $-n(p)$ in (4.6)]. This has a novel consequence for our metrics $\stackrel{\circ}{g}$ (to be introduced shortly below): that instead of having uniqueness for $g_{+}$, we necessarily find a non-trivial asymptotic condition on the scri of the ambient metric $g_{+}$for $\left.\stackrel{\circ}{g}\right|_{M}$. The last property of $n$ in the enumeration above implies that the FM metrics appear in this context as the $p \rightarrow+\infty$ limit of our geometry.

The form of the ambient metric (4.6) implies that the original (asymptotic splitting) form of the density $\rho=$ $y^{p^{\prime}} \sum_{i=0}^{\infty} c_{i} y^{i / s}$, with $p^{\prime}=m^{\prime} / l^{\prime} \in \mathbb{Q}, m^{\prime} \in \mathbb{Z}, l^{\prime} \in \mathbb{N}$, becomes, in terms of $r$,

$\rho(r)=r^{s m^{\prime}} \sum_{i=0}^{\infty} c_{i}^{\prime} r^{i l^{\prime}}$,

and therefore it takes the generic form (for $p \neq 1$ )

$\rho_{+}=w^{z} \theta(w), \quad z=\frac{p^{\prime}}{1-p} \frac{l^{\prime}}{l}, \quad \theta$ smooth.

When $p=1$, we find, $\rho(w)=e^{m^{\prime} w} \theta(w)$, and then the transformation $w \rightarrow 1 / w$ makes the density a $C^{\infty}$ function, if we define $\rho(0)=0$.

\section{The conformal infinity of the ambient metric}

To complete the construction of the ambient metric of the previous section, we discuss the two cases we left previously, namely, when $p=1$ and when $p \in(0,1)$. To proceed, on $V=M \times \mathbb{R}$, we define the product metric

$\stackrel{\circ}{g}=\omega^{2} g_{+}$,

where $\omega>0$ on $V \backslash M$, and zero on $M$. (Later in this section, we choose $\omega=w^{n}$, but this is not important presently.) We will shortly see that in this form, the metric $\stackrel{g}{g}$ in Eq. (5.1) represents the regular (see below) conformal infinity of $g_{+}$, and justifies step 6 of Sect. 3. This means that when $g_{+}$ blows up to $+\infty$ at points of the boundary $M$, the conformal factor $\omega$ tends to zero and so is responsible for a conformal 
squashing of $g_{+}$producing a finite $\stackrel{\circ}{g}$ at infinity, whereas when $g_{+}$crunches to zero, $\omega$ blows up to infinity, again making $\stackrel{\circ}{g}$ finite there. All results above correspond to $\omega \rightarrow 0$ at the conformal infinity of $g_{+}$, since $g_{+}$diverged there, but when $p=1$, or $p \in(0,1)$, we shall find that $\omega \rightarrow \infty$.

When $p=1$, the ambient metric will read $g_{+}=$ $e^{2 w}\left(\sigma^{2}(w) g_{4}\left(x^{\mu}\right)+d w^{2}\right)$, where $w / s=\ln r$, and so at the conformal boundary (i.e., as $r \rightarrow 0$ ), the conformal factor of the ambient 5-geometry in Eq. (5.1), $\omega^{2}=e^{-2 w}$, will tend to $+\infty$ at $\mathscr{I}$. Similarly, when $p \in(0,1)$, then $-n>0$, and the ring metric becomes $\stackrel{\circ}{g}=w^{n} g_{+}$, which means that the conformal factor $\omega=w^{n}, n<0$, diverges at the boundary ( $w \rightarrow 0$ now). We therefore see that both these cases are exceptional in the sense that the conformal factor $\omega$ diverges at the boundary points, instead of tending to zero there as it does for all other $p$ values. Hence, it appears that when $p \in(0,1]$, the behavior of $\omega$ which describes the scaling between the two metrics $\stackrel{\circ}{g}$ and $g_{+}$is somehow inverted, so that $\omega$ becomes its reciprocal, $\omega \rightarrow 1 / \omega$.

Since the exponent $p$ is generically [20-23] a function of the equation-of-state parameter $\gamma$ in the 5-fluid equation of state $P=\gamma \rho$ in Eqs. (2.1)-(2.3), we may interpret the above situation by adopting the view that $\omega$ (or its inverse) as a function of $\gamma$ is not smooth. In general, $\omega$ is also a real-valued function defined on the set $M \times \mathbb{R}$, so we may write $\omega=\omega_{\gamma}\left(x^{\mu}, w\right)$, and think of the conformal factor $\omega$ as a 1-parameter family of functions on $V$. The description of the conformal boundary $M$ in terms of properties of the family $\omega_{\gamma}$ is a more complicated problem, currently under investigation.

Having constructed the ambient metric $g_{+}$given by (4.6), steps 6,7 of the construction now follow. For, if on $V=$ $M \times \mathbb{R}$ we define the product metric

$\stackrel{\circ}{g}=w^{n} g_{+}=\sigma^{2} g_{4}+d w^{2}$,

and since $g_{4}$ is non-singular, we find that

$\operatorname{det} \stackrel{\circ}{g} \neq 0, \quad \sigma^{2}(w)=c_{0}+c_{1} w+\cdots$,

so the metric (5.2) is manifestly regular at $w=0$. Also, the metric $\left.\left(w^{n} g_{+}\right)\right|_{M}=\sigma^{2}(w) g_{4}$ is clearly a metric belonging in the conformal class $\left[g_{4}\right]$ (albeit one with smooth conformal factor not depending on $x^{\mu}$ but only on $w$ ). Thus a conformal infinity exists for the ambient metric $g_{+}$given by (4.6), and step 6 follows.

For the conformal compactness of the ambient metric $g_{+}$ of step 7 of Sect. 3, we first note that since $\sigma(w)$ is a smooth function, we find that (5.2) extends smoothly everywhere in the ambient spacetime $V$. As we already discussed, the metric $\stackrel{\circ}{g}$ is non-unique in the sense that any function $\omega(w)$ with the required properties will do. Here, we have chosen $\omega=w^{n}$, but this choice is not unique.

To show the non-degeneracy part of the property of conformal compactness, we proceed as follows. Since $\stackrel{\circ}{g}$ is a spacetime metric, it will have indefinite signature. Because its restriction to the first factor, $\left.\stackrel{\circ}{g}\right|_{M}$, that is, the pullback $j^{*}(\stackrel{\circ}{g})$ by the inclusion map $j: M \rightarrow V$, is given by the form

$\left.\stackrel{\circ}{g}\right|_{M}=\sigma^{2}(w) g_{4}$,

it follows that $\left.\stackrel{\circ}{g}\right|_{M}$ will be a well-defined metric on $M$ if and only if $g_{4}$ is non-degenerate, so that $\stackrel{\circ}{g}$ sustains the same signature also on $M$. Now, the Penrose construction [26] utilized in step 2 generally guarantees the non-degeneracy of the 'nice' metric $g_{4}$. In general, the non-degeneracy of the metric $\left.\stackrel{\circ}{g}\right|_{M}$ dictates that $g_{4}$ should not become degenerate at any point of $M$, in any case, we view (5.4) as a kind of compatibility condition. We shall have to say more on this in Sect. 8.

To prepare for step 8 and the asymptotic conditions treated next, we end this section with the following remarks. From the results up to now, it follows that for two conformally related 4-metrics of $M, g_{1}=\Omega^{2} g_{2}$, the difference of their ambient metrics on $M$ is at best (meaning even for $w=0$ ) equal to $\left.\stackrel{\circ}{g}_{1}\right|_{M}(0)-\left.\stackrel{\circ}{g}_{2}\right|_{M}(0)=c_{1} g_{1}-c_{2} g_{2}$, with $c_{1}, c_{2}$ being the well-defined, nonzero constants $c_{i}=\sigma_{i}^{2}(0), i=1,2$. So it is not obviously zero, and uniqueness of the ambient metric cannot really follow by simply quoting the case of the FG metrics (cf. [24], chapters 3, 4). This is because in their metrics in normal form, $g_{+}=w^{-2}\left(g_{w}+d w^{2}\right)$, the 1-parameter family $g_{w}$ of metrics on $M$ does not necessarily have the same form as our explicitly constructed $\sigma^{2}(w) g$. In any case, they do not have a fluid in the ambient space like we do, only a cosmological constant, and so an adaptation of their uniqueness results to our case is not straightforward. We also note that while the property of being an Einstein space, i.e., $\operatorname{Ric}\left(g_{4}\right)+\lambda g_{4}=0$, is preserved under a conformal rescaling with constant factor, $g_{4} \rightarrow c g_{4}$, this is not so for solutions of the Einstein equations with a fluid source.

\section{The asymptotic conditions}

In this section we focus on proving step 8 of Sect. 3, in particular, we show that the ambient metric of any 4-metric in the conformal class of a given 4-metric on $M$, although not unique, has a 'universal' conformal infinity in the sense of giving a hugely simplified geometry in comparison to the initial 4-metric on $M$ it arose from.

In particular, we show that if we take two 4-metrics $g_{1}, g_{2}$ on $M$ belonging to the same conformal class, that is, such that there is a smooth conformal factor $\Omega\left(x^{\mu}\right)$ with $g_{1}=\Omega^{2} g_{2}$, then the $M$-restriction of the ambient metric of $g_{2}$ at $w=0$, that is, the metric $\left.\stackrel{\circ}{g}_{2}\right|_{M}(0)$, equals a nonzero constant times the 'good' metric $g_{1}\left(x^{\mu}\right)$. We may think of $g_{1}$ as a 'good' metric as in step 2 and $g_{2}$ as a 'bad' metric of step 1 -i.e., one blowing up at $\Omega=0$, the scri of $M, \mathscr{I}_{M}$. 
For two such 4-metrics $g_{1}, g_{2}$ of $M$, their ambient metrics on $M \times \mathbb{R}$ are given as the product metrics

$\stackrel{\circ}{g}_{i}(w)=\sigma_{i}^{2}(w) g_{i}\left(x^{\mu}\right)+d w^{2}, \quad i=1,2$.

Since $g_{2}\left(x^{\mu}\right)$ would possibly become infinite at points of its conformal boundary, we exclude such points $x^{\mu}$ from the right hand side of (6.1) for $\stackrel{\circ}{g}_{2}(w)$. Then the restrictions to $M$ of the two ambient metrics in (6.1) will differ by the symmetric 2-tensor $\hat{g}(w)=\left.\stackrel{\circ}{g}_{1}\right|_{M}(w)-\left.\stackrel{\circ}{g}_{2}\right|_{M}(w)$ where,

$\hat{g}(w)=g_{2}\left(\sigma_{1}^{2}(w) \Omega^{2}\left(x^{\mu}\right)-\sigma_{2}^{2}(w)\right)$,

and the problem becomes one of deciding how bad this difference can really be. This difference measures how far the ambient metric of the 'bad' metric $g_{2}$ is from that of the good metric $g_{1}$. If this difference were not near $g_{1}$, then the ambient construction could not really be considered as something worth doing. On the other hand, if this difference were zero then the ambient metric would be the one and only metric for all 4-metrics in the conformal class of a given 4-metric on $M$. This is the situation with the FG metrics [24]. Below, we show that in our construction this difference, although nonzero, becomes a constant multiple of $g_{1}$, and so the ambient metric of any $g_{2}$ in the conformal class of $g_{1}$ corresponds to a great simplification over the original situation of having the two metrics $g_{1}, g_{2}$ on $M$. We note that the difference $\hat{g}(w)$ is not a well-defined metric on $V$ because it is degenerate, and so its projection to $M$ is not defined. Only first restricting the two ambient metrics (6.1) to $M$ as in (5.4) and then taking their difference, produce a non-degenerate, well-defined tensor field on $M$ (see also below).

Setting $w=0$ in Eq. (6.2), the difference will be,

$\left.\hat{g}\right|_{M}=g_{2}\left(c_{0} \Omega^{2}\left(x^{\mu}\right)-c_{0}^{\prime}\right)$,

where $c_{0}, c_{0}^{\prime}$ are the first constants in the formal series of the two ambient metrics. Since these constants are nonzero and uniquely determined by the method of asymptotic splittings, while $\nabla_{g_{1}} \Omega \neq 0$, as this is assumed in the conformal method [26] so that $\Omega$ is not a constant on $M$, the term in the brackets in Eq. (6.3) is not zero. Therefore, the function in the brackets in Eq. (6.2) is not identically zero. Hence, we may regard this $\hat{g}(w)$ from Eq. (6.2) as a well-defined, 2-tensor living on the original 4-manifold $M$, which has the variable $w$ as a parameter. In this sense, $\hat{g}(w)$ represents a 1-parameter family of 4-dimensional tensor fields on $M$ depending on $w$, and so we can take successive derivatives of $\hat{g}(w)$ in Eq. (6.2) with respect to $w$ and then set $w=0$.

Now, we know from the method of asymptotic splittings that at finite orders in the series $\sigma_{1}, \sigma_{2}$, there appear arbitrary constants as coefficients precisely in those terms in the series where the exponents equal the eigenvalues of the Kowalevskaya matrix. So if the order $i=i_{0}$ is such that $i_{0} / s=\rho_{i_{0}}$, where $\rho_{i_{0}}$ is an eigenvalue of the Kowalevskaya matrix (for the 5-dimensional Einstein equations with the fluid source we solved to find $g_{+}$), then the corresponding coefficient $c_{i_{0}}$ in front of that term in the series $\sigma(w)=$ $\sum_{i}^{\infty} c_{i} w^{i}$ will be an arbitrary constant. We know that arbitrary constants appear in the terms with exponents equal to those eigenvalues of the Kowalevskaya matrix (the so-called 'K-exponents') which have strictly positive real parts (corresponding to the unstable eigenspaces), and this property is not only restricted to the case of the Puiseux series (i.e., e rational exponents), but continues to hold for the general $\Psi$-series containing log terms. Provided we set the arbitrary coefficients corresponding to the negative eigenvalues equal to zero, the resulting series developments always exist as convergent sums [31].

We note in passing that our restriction to Puiseux series from the beginning in this work was because we looked for general solutions to the 5-dimensional Einstein equations with fluid sources in [20-23] in an effort to answer the question whether or not generic solutions of the system were singular, that is, whether singularities were a typical feature of all solutions in 5-dimensions. Had we instead started with a $\Psi$-series ansatz containing log terms and set to zero some of the arbitrary constants in the supposed solutions at the end would produce only particular solutions, since we would have sacrificed some of the arbitrary constants, and we could not contribute usefully to that search. However, for the purposes of the arguments in the current work, we only need some (in particular, the positive) arbitrary constants to be nonzero, not all, and therefore we conclude that all arguments presently would continue to be valid had we considered a $\Psi$-series instead of simple Puiseux expansions from the beginning.

We can now differentiate Eq. (6.2) with respect to $w$ precisely $i_{1}$ times, where $i_{1}$ is the order where the first arbitrary coefficient $c_{i_{1}}^{\prime}$ in the expansion $\sigma_{2}^{2}(w)$ associated with the 'bad' metric $g_{2}$ appears. Then Eq. (6.2) becomes

$$
\begin{array}{r}
\frac{\partial^{i_{1}} \hat{g}}{\partial w^{i_{1}}}(w)=g_{2}\left(\left(c_{i_{1}}+c_{i_{1}+1} w^{i_{1}+1}+\cdots\right) \Omega^{2}\left(x^{\mu}\right)\right. \\
\left.-\left(c_{i_{1}}^{\prime}+c_{i_{1}+1}^{\prime} w^{i_{1}+1}+\cdots\right)\right) .
\end{array}
$$

Here $c_{i_{1}}$ is fixed but the constant $c_{i_{1}}^{\prime}$ is arbitrary. This is so because the Kowalevskaya matrices corresponding to the metrics $g_{1}, g_{2}$ do not in general have the same eigenvalues, so that one should not expect that both constant coefficients $c_{i_{1}}, c_{i_{1}}^{\prime}$ will be arbitrary at the same order $i_{1}$. Setting now $w=0$, we find that

$\frac{\partial^{i_{1}} \hat{g}}{\partial w^{i_{1}}} \|_{w=0}=g_{2}\left(c_{i_{1}} \Omega^{2}\left(x^{\mu}\right)-c_{i_{1}}^{\prime}\right)$.

In this equation, unlike Eq. (6.3), we are allowed to set the arbitrary constant $c_{i_{1}}^{\prime}=0$. Then we find the following asymptotic condition: 
$\frac{\partial^{i_{1}} \hat{g}}{\partial w^{i_{1}}} \|_{w=0}=c_{i_{1}} g_{1}\left(x^{\mu}\right), \quad$ on $\quad M$,

where $g_{1}$ is the 4-dimensional metric on $M$ with 'nice' properties. We note the following remarkable fact: Although from the beginning of this section and up to and including Eq. (6.5) we had to exclude the singular boundary points where the 'bad' metric $g_{2}$ would diverge, Eq. (6.6) holds even at those singular points because $g_{1}$ is perfectly regular there.

Now suppose that the Kowalevskaya matrix corresponding to the 5-dimensional Einstein equations for ambient metric $g_{2,+}$ of the 'bad' metric $g_{2}$ has exactly $k$ positive eigenvalues $\rho_{i_{1}}=i_{1} / s, \cdots \rho_{i_{k}}=i_{k} / s$. Following the procedure above, we will end up with $k$ asymptotic conditions, one for each one of the $k$ derivatives of various orders of the metric $\hat{g}$, evaluated at $w=0$ :

$\frac{\partial^{i_{1}} \hat{g}}{\partial w^{i_{1}}}\left\|_{w=0}=c_{i_{1}} g_{1}, \ldots, \frac{\partial^{i_{k}} \hat{g}}{\partial w^{i_{k}}}\right\|_{w=0}=c_{i_{k}} g_{1}, \quad$ on $M$.

This is the set of the required asymptotic conditions we need to determine $\hat{g}$. For, since we know that $k$ derivatives of that function at 0 are all constants times the metric $g_{1}$, we conclude that

$\left.\hat{g}\right|_{M}(w)=e^{a w} g_{1}\left(x^{\mu}\right), \quad a=\sum a^{j} c_{j}, \quad j=i_{1}, \cdots, i_{k}$.

The $k$ unknown constant coefficients $a^{j}$ are found by solving the nonlinear algebraic system

$$
\left(\sum a^{j} c_{j}\right)^{i_{1}}=c_{i_{1}}, \ldots,\left(\sum a^{j} c_{j}\right)^{i_{k}}=c_{i_{k}} .
$$

This system always has a solution for $a$ in the complex field. We note that Eq. (6.8) holds also for the singular boundary of the 'bad' metric $g_{2}$ for the same reason as before, namely, because $g_{1}$ is perfectly regular at these points.

We may think of the result (6.8) as valid in a small, local neighborhood around $w=0$, because the extension of the conformal boundary of the 4-metric $g_{1}$ to $V$ is regular since we proved that $\stackrel{\circ}{g}_{1}$ is regular in $V$. There the difference $\left.\hat{g}\right|_{M}(w)$ is a well-behaved multiple of $g_{1}$, the factor depending only on $w$. Setting $w=0$ in Eq. (6.8), we find that

$\left.\stackrel{\circ}{g}_{1}\right|_{M}(0)-\left.\stackrel{\circ}{g}_{2}\right|_{M}(0)=g_{1}$,

essentially a condition on the conformal infinity of the ambient metric $g_{2,+}$ of the 'bad' 4-dimensional metric $g_{2}$. It means that the conformal infinity of the 5-metric $g_{2,+}$ is equipped with the metric $\left.\stackrel{\circ}{g}_{2}\right|_{M}$, which differs from the 'nice' conformal infinity $\left.\stackrel{\circ}{g}_{1}\right|_{M}$ by the 'nice' metric $g_{1}$, and so it cannot be very 'wild', it will be a rescaling of the 'good' metric $g_{1}$. This is true also for the points on the singular 4-boundary of the metric $g_{2}$ for reasons discussed above.

This $w$-rescaling of $g_{1}$ from Eq. (6.8) will have the form $\sigma_{1}^{2}(w)-e^{a w}$, where $\sigma_{1}^{2}$ is a convergent, formal power series expansion for the ambient metric $g_{1,+}$ of the 'good' metric $g_{1}$, with $w$ taking values in a small neighborhood of the form $(-\epsilon, \epsilon)$ around 0 . At 0 , it will read $c_{0}-1$, and so we find

$\left.\stackrel{\circ}{g}_{2}\right|_{M}(0)=\left(c_{0}-1\right) g_{1}\left(x^{\mu}\right)$.

We note that when $c_{0}$ turns out to be 1 (as could result by the application of the method of asymptotic splittings), we may use the freedom to multiply the right hand side of Eq. (6.8) by a different integration constant of the form $C=e^{b}$, which was chosen equal to 1 above. The system for $a=$ $\sum a^{j} c_{j}+b$ would then solve as before, but with $b$ added inside the brackets in the left hand sides of (6.9), namely, we will have the system,

$$
\left(\sum a^{j} c_{j}+b\right)^{i_{1}}=c_{i_{1}}, \ldots,\left(\sum a^{j} c_{j}+b\right)^{i_{k}}=c_{i_{k}} .
$$

Hence the constant in the right hand side of (6.11) may be assumed to be nonzero. When this factor is positive, the constant rescaling (homothety) of the metric $g_{1}$ preserves the causal character of curves on $M$, while when it is negative we have the so-called metric-reversing [33]. In this case, all geometric notions associated with the homothetic transformation remain the same, however, the causal character of timelike and spacelike vector fields is reversed, while null ones remain so. (In any case, the sign of the constant factor is controlled by the choice of $b$.)

Equation (6.11) is then, in our context, the condition replacing uniqueness of the Fefferman-Graham ambient metric. We call this 'the asymptotic condition' because it is one valid on the conformal infinity $M$ of the ambient space $V$ after taking the limit $w \rightarrow 0$. We have shown the following result.

Theorem 6.1 A4-metric $g$ in the conformal class of a 'good' 4-metric $g_{4}$ on $M$ has an ambient metric $g_{+}$which satisfies the 5-dimensional Einstein equations with a fluid source and has conformal infinity $\left(\mathscr{I}_{g_{+}},\left.\stackrel{\circ}{g}\right|_{M}\right)$ described as a constant rescaling of $g_{4},\left.\stackrel{\circ}{g}\right|_{M}=c g_{4}$. Any two conformally related 4-metrics on $M, g_{1}=\Omega^{2} g_{2}$, can be chosen to have ambient metrics differing by $\left.\stackrel{\circ}{g}_{1}\right|_{M}(0)-\left.\stackrel{\circ}{g}_{2}\right|_{M}(0)=g_{1}$.

This concludes the discussion of our ambient construction. In the next two sections, we examine two important implications of the ambient geometric structures studied so far.

\section{Conformal structure and singularities}

Suppose that we start in step 1 of the ambient algorithm with a spacetime $\left(M, g_{\mathrm{IN}}\left(x^{\mu}\right)\right)$ which in step 2 gives a conformally related 4-dimensional metric $g_{4}=\Omega^{2} g_{\mathrm{IN}}\left(x^{\mu}\right)$. Then it follows from the work we did in previous sections that these 
two metrics have ambient metrics with the following property. To $g_{4}$, there is an ambient 5-metric $g_{+}$satisfying the 5-dimensional Einstein equations with a fluid source such that its conformal infinity is described by the 4-dimensional metric $\left.\stackrel{\circ}{g}\right|_{M}$ that belongs to the conformal class $\left[g_{4}\right]$ and is a constant $w$-rescaling of $g_{4}$. Similarly, we can construct the ambient metric of $\left(M, g_{\text {IN }}\left(x^{\mu}\right)\right)$ (or of any other metric in the conformal geometry $\left.\left[g_{4}\right]\right)$ which will satisfy the 5-dimensional Einstein equations with a fluid source and the metric $\left.\stackrel{\circ}{g}_{\mathrm{IN}}\right|_{M}$, will be a different $w$-rescaling of $g_{4}$. The difference of the two ambient metrics $\left.\stackrel{\circ}{g}_{\mathrm{IN}}\right|_{M}$ and $\left.\stackrel{\circ}{g}_{4}\right|_{M}$ corresponding to the two conformally related boundary metrics $\stackrel{\circ}{g}_{\text {IN }}$ and $g_{4}$ equals the initial metric $g_{4}$.

Therefore, since for a given $g \in\left[g_{4}\right],\left.\stackrel{\circ}{g}\right|_{M}$ is an honest metric we may imagine our 4-dimensional universe described as the conformal infinity of the ambient 5-metric $g_{+}$. In this case, the properties of our 4-dimensional world would be dictated by the 5-dimensional ambient spacetime and the ambient metric $g_{+}$satisfying the 5-dimensional Einstein-fluid equations. Then, various fundamental cosmological questions acquire novel meanings: Will there be any singularities in the 4-dimensional universe $\left(M,\left.\stackrel{\circ}{g}\right|_{M}\right)$ ? Is cosmic censorship valid on $\left(M,\left.\stackrel{g}{g}\right|_{M}\right)$ ? What is the behavior of the various fields living on the ambient space at their conformal infinity? What is the behavior of the entropy on $M$, and how can we distinguish initial from final singularities there? What is the relation of the properties of $(M,[g])$ to quantum gravity? Are there any observational tests that point to the structure of $\left(M,\left.\stackrel{\circ}{g}\right|_{M}\right)$ ?

For the ambient 5-spacetime $V$, these are all deep and difficult questions, and so the reader may wonder what we have gained with our construction. In general, given a conformal structure on the boundary 4-spacetime $\left(M,\left[g_{4}\right]\right)$ as we have done above, the ambient cosmologies will not of course have a form like the one we assumed above, namely, $a^{2}(w) g_{4}+d w^{2}$, since we do not know the global solution to the 5-dimensional Einstein equations with fluid sources in the ambient spacetime (and even if we did, it would probably be exceedingly difficult to extract any information by elementary arguments).

However, suppose that we are not interested in what happens everywhere in the ambient spacetime $V=M \times \mathbb{R}$, but only care about the physics on $M$. In this paper, we have constructed the notion of an ambient cosmology by starting from a conformal structure on $M$, and found that there is an ambient metric $g_{+}$described in terms of formal power series solutions locally in $V$ about $M$ of the 5-dimensional Einstein equations with fluid sources in $V$. Corresponding to the conformal structure on $M$, the ambient metric $g_{+}$on $V$ has conformal infinity that inherits the asymptotic properties of a 'nice' metric $g_{4}$ in the conformal $M$-geometry and is described as a homothetic multiple of $g_{4}$.
Therefore in this model, all physical properties of $M$ are captured and imprinted on it by its conformal structure $\left(M,\left[g_{4}\right]\right)$ and the metric $\left.\stackrel{g}{g}\right|_{M}$, which is in turn determined by the ambient metric formal series solutions about $M$ of the 5dimensional Einstein equations with fluid sources. One may ask: How do the asymptotic properties of the ambient metric $g_{+}$and thermodynamic quantities such as the density $\rho_{+}$of the 5-dimensional solutions affect the behavior on $M$ ? For example, if $\rho_{+}$diverges on points of $M$, or everywhere on $M \times\{0\}$, how will such a behavior on $V$ affect that on $M$ ? As we have shown in previous sections, the form of the $M$ restriction of the ambient metric $\stackrel{\circ}{g}=\sigma(w) g_{4}$, for a $g \in\left[g_{4}\right]$, is determined by the smooth function $\sigma(w)$ and the original 4-metric $g_{4}$, so that it reads, $\stackrel{\circ}{g}=\left(c_{0}+c_{1} w+\cdots\right) g_{4}$, with the constants determined by the 5 -dimensional solutions of the Einstein equations with the fluid source. Hence, the possibly diverging behavior of the 5-dimensional density $\rho_{+}$at $M$, passes on to the metric $\left.\stackrel{\circ}{g}\right|_{M}$ on $M$ only through the constants $c_{0}, c_{1}, \ldots$, in the sense that the different possible behaviors of $\rho$ would just readjust the values of these constants in a new $\left.\stackrel{\circ}{g}\right|_{M}$.

We may therefore conclude that any singularities present in the metric $\left.\stackrel{\circ}{g}\right|_{M}$ will be those remaining in $g_{4}$ after the conformal 'cleaning' of $g_{\mathrm{IN}}\left(x^{\mu}\right)$ in step 2. No other new ones will arise from the ambient spacetime $V$ following our construction. What are the constraints on $g_{4}$ ? The metric $g_{4}$ is seen to satisfy two conditions: The first is that it is constructed as in step 2 following the application of the conformal method [26] in step 1, and the second is that it must comply with the basic condition Eq. (6.11). This can give an incompatible constraint on $\left.\stackrel{\circ}{g}\right|_{M}$ only when $g_{4}$ (and, consequently, any homothetic multiple of it) becomes degenerate somewhere on $M$. This is discussed more fully in the next section.

Compared with the standard situation described in general relativity as having the initial metric $\left(M, g_{\mathrm{IN}}\left(x^{\mu}\right)\right)$ satisfying the 4-dimensional Einstein equations with some sources, our situation described by the metric $\left.\stackrel{\circ}{g}\right|_{M}$ induced on $M$ (and belonging in its conformal structure as above) constitutes a considerable improvement. The 4-metric $\left.\stackrel{\circ}{g}\right|_{M}$ imposed on $M$ by the 5 -dimensional ambient model discussed above inherits the properties of the 'nice' metric $g_{4}$ (which in turn cannot be a solution of the same 4-dimensional Einstein equations satisfied by $g_{\mathrm{IN}}\left(x^{\mu}\right)$ because it is related conformally to the latter), while it does not receive any new singularities from the 5-dimensional ambient metric because it lies in its conformal infinity as described in this paper.

\section{Non-degeneracy and cosmic censorship}

In this work we have shown that there is a relation between the conformal structure of the boundary geometry and the 5-dimensional ambient metric construction. The ambient 
metric leads to the asymptotic condition (6.11), $\left.\stackrel{\circ}{g}\right|_{M}=c g_{4}$, satisfied by the metrics $g_{4}$ and $\left.\stackrel{\circ}{g}\right|_{M} \in\left[g_{4}\right]$, and this imposes a compatibility condition on the two homothetically related metrics. In particular, since $\left.\stackrel{\circ}{g}\right|_{M}=\sigma g$ is by its construction non-degenerate on $M$, this means that $c g_{4}$ must also be a non-degenerate metric. We note that the latter is obtained by finding the conformal infinity of the ambient metric corresponding to the 'nice' metric $g_{4}$, and then any metric $g$ in the conformal class of $g_{4}$ with have this form. Thus, nondegeneracy of the $g_{4}$ metric in step two of the ambient algorithm, is an immediate implication of the ambient construction on the conformal structure of $M$. (We note that metric reversal does not produce degeneracy on $g_{4}$.) Is it possible that $g_{4}$, in the process of step 2, develops singularities or other structures that make the ambient condition (6.11) an incompatible constraint for the ambient construction? This is a question about the structure of the singular boundaries of the two metrics in (6.11), which we address below.

We first note that a singular metric $g_{4}$ does not necessarily make the 5-dimensional ambient metric $g_{+}$(or the metric $\stackrel{\circ}{g}$ ) degenerate, unless it becomes null or timelike somewhere. Therefore if a $g_{4}$ develops a singularity that is spacelike everywhere, then $\stackrel{\circ}{g}$ will still be non-degenerate when restricted on the boundary. (For example, such will be the case for the singular part of the horizon in non-static coordinates for the Schwarzschild black hole, while the null part is regular.)

In general, non-degeneracy of the 5-dimensional metrics $\stackrel{\circ}{g}$ or $g_{+}$means that they do not become degenerate on important subspaces of the ambient spacetime, especially, this must be so at $y=0$, so we require that these metrics, when restricted on the boundary spacetime $M$, must not become degenerate. Since the signature of the 5-dimensional metrics is $(1,3+1)$ (meaning one - and four + 's), non-degeneracy implies that on the conformal boundary $M$ the restriction of the metric $g_{+}$ must retain its signature $(1,4)$. Since the boundary spacetime signature is $(1,3)$, we find that the boundary 3 -space signature should not globally change from +++ to something else, that is, to null -++ , or to timelike --- , because such a change would make the 5-dimensional metric degenerate (in the sense that a null surface could then form in the ambient spacetime).

We propose that the choice of metric in the conformal class $\left[g_{4}\right]$ in step 2 of the ambient procedure of Sect. 2, must be made such that it does not spoil the non-degeneracy of the $\stackrel{\circ}{g}$ metric when restricted along the boundary $M$. As discussed above, the only way then left for which the 5-dimensional ambient metric will lose its non-degeneracy on $M$ is when a timelike or null hypersurface forms somewhere in $\left.\stackrel{\circ}{g}\right|_{M}$, that is, when there are naked points at infinity on the boundary spacetime. This then would make the ambient cosmology $\left.\stackrel{\circ}{g}\right|_{M}$ degenerate, and the difference $\left.\hat{g}\right|_{M}$ in (6.10) will not make sense, a contradiction because as we showed this dif- ference equals a rescaling of a well-defined spacetime metric. Therefore it seems that a choice must be made of those metrics $g_{4}$ in step 2 of the ambient procedure that respect cosmic censorship. $^{4}$

Conversely, the absence of naked singularities which follows from the validity of the asymptotic (in the sense of being valid on $\mathscr{I}_{V}$ ) condition (6.11) of the ambient cosmology has important implications. For example, it follows that a naked singularity may not be the end product of the process of Hawking evaporation of a black hole through thermal radiation. In this case, future null infinity will generically meet the vertical line coming out of the spacelike singularity of the black hole due to the evaporation (compare the Penrose diagrams in Figs. 3 and 5 of Ref. [36]), thus allowing material from inside the spacelike singularity to be seen by an observer sitting at infinity. This is sometimes interpreted, as is well known, as a possible violation of cosmic censorship at the quantum level, a complete loss of predictability in a quantum treatment of black holes [37], and it is also intimately related to the possible loss of information connected with the inevitable increase of entropy during this process [38].

Note added in proof: After the completion of this work, we realized that there are certain analogies with some of the ideas of conformal cyclic cosmology [39]. The ideas of masslessness near the singularities and a possible universal decay of all particle masses asymptotically in [39] may also be applicable here and associated with the crucial role played by conformal (instead of Riemannian) geometry in the 4-dimensional conformal infinity of the 5-dimensional ambient metric. However, in our work, we only have 5dimensional Einstein equations with sources for the ambient metric. Another central characteristic implication of our work is that the existence of black holes and the validity of cosmic censorship in the 4-dimensional boundary spacetime seem to be intimately connected with the ambient construction taking place in the extra dimension, because they appear as properties of its conformal infinity. We believe it is an interesting question whether the approach developed here allows or even requires any cyclicity in the 4-dimensional conformal manifold.

\footnotetext{
4 The only other possibility is that the non-degeneracy of the ambient metric is spoiled when spherical symmetry is violated, for example when a suitable observer who falls into a black hole in an exact Kerr metric with $a<m$ (with $m, a$ constants, $m$ being the mass and $m a$ the angular momentum as measured from infinity), to whom the singularity is actually naked. However, there are reasons to believe that such a situation inside the event horizon is actually unstable, cf. [34], p. 234, and generically perturbed Kerr solutions will always develop a spacelike singularity in the neighborhood of its Cauchy horizon. A spacelike character of cosmological singularities is also supported by the BKL studies, cf. [35] and references therein.
} 
Acknowledgments We thank Ioannis Bakas, Manolis Floratos, and Kyriakos Papadodimas for useful discussions, and an anonymous referee for many useful and constructive comments which helped to present this work in a more comprehensive way.

Open Access This article is distributed under the terms of the Creative Commons Attribution License which permits any use, distribution, and reproduction in any medium, provided the original author(s) and the source are credited.

Funded by $\mathrm{SCOAP}^{3}$ / License Version CC BY 4.0.

\section{References}

1. P. Hôrava, E. Witten, Nucl. Phys. B460, 506 (1996); Nucl. Phys. B475, 94 (1996)

2. N. Arkani-Hamed, S. Dimopoulos, G. Dvali, Phys. Lett. B429, 263 (1998)

3. I. Antoniadis, N. Arkani-Hamed, S. Dimopoulos, G. Dvali, Phys. Lett. B436, 257 (1998)

4. P. Binetruy, C. Deffayet, D. Langlois, Nucl. Phys. B565, 269-287 (2000)

5. G. 't Hooft, Dimensional reduction in quantum gravity, THU93/26. arXiv:gr-qc/9310026

6. J. Maldacena, Adv. Theor. Math. Phys. 2, 231 (1998)

7. E. Witten, Adv. Theor. Math. Phys. 2, 253-291 (1998)

8. R. Penrose, Phys. Rev. Lett. 14, 57 (1965)

9. S.W. Hawking, R. Penrose, Proc. R. Soc. Lond. A314, 529 (1970)

10. L. Randall, R. Sundrum, Phys. Rev. Lett. 83, 3370 (1999)

11. L. Randall, R. Sundrum, Phys. Rev. Lett. 83, 4690 (1999)

12. N. Arkani-Hamed, S. Dimopoulos, N. Kaloper, R. Sundrum, Phys. Lett. B480, 193-199 (2000). arXiv:hep-th/0001197

13. S. Kachru, M. Schulz, E. Silverstein, Phys. Rev. D 62, 085003 (2000). arXiv:hep-th/0002121

14. S.S. Gubser, Adv. Theor. Math. Phys. 4, 679-745 (2000). arXiv:hep-th/0002160

15. S. Forste, Z. Lalak, S. Lavignac, H.P. Nilles, Phys. Lett. B 481, 360 (2000). arXiv:hep-th/0002164

16. S. Forste, Z. Lalak, S. Lavignac, H.P. Nilles, JHEP 0009, 034 (2000). arXiv:hep-th/0006139

17. S. Forste, H.P. Nilles, I. Zavala, JCAP 1107, 007 (2011). arXiv:1104.2570 [hep-th]

18. R. Penrose, J. Astrophys. Astr. 20, 233-248 (1999)

19. S. Cotsakis, J.D. Barrow, J. Phys. Conf. Ser. 68, 012004 (2007). arXiv:gr-qc/0608137
20. I. Antoniadis, S. Cotsakis, I. Klaoudatou, in Proceedings of the MG11 Meeting, ed. by H. Kleinert, R.J. Jantzen, R. Ruffini (World Scientific, 2008), pp. 2054-6. arXiv:gr-qc/0701033

21. I. Antoniadis, S. Cotsakis, I. Klaoudatou, Class. Q. Grav. 27, 235018 (2010). arXiv: 1010.6175

22. I. Antoniadis, S. Cotsakis, I. Klaoudatou, Fortschr. Phys. 61, 20-49 (2013). arXiv: 1206.0090

23. I. Antoniadis, S. Cotsakis, I. Klaoudatou, Eur. Phys. J. C 74, 3192 (2014). arXiv:1406.0611

24. C. Fefferman, C. Robin Graham, The Ambient Metric (Princeton University Press, Princeton, 2012). arXiv:0710.0919

25. K. Skenderis, Class. Q. Grav. 19, 5849-5876 (2002). arXiv:hep-th/0209067

26. R. Penrose, W. Rindler, Spinors and Space-Time, vol. 2 (Cambridge University Press, Cambridge, 1986), chap. 9

27. S.W. Hawking, G.F.R. Ellis, The Large Scale Structure of SpaceTime (Cambridge University Press, Cambridge, 1973)

28. R.H. Dicke, The Theoretical Significance of Experimental Relativity (Gordon and Breach, New York, 1964)

29. Y. Fujii, K. Maeda, The Scalar-Tensor Theory of Gravitation (Cambridge University Press, Cambridge, 2003)

30. M. Gasperini, Elements of String Cosmology (Cambridge University Press, Cambridge, 2007)

31. A. Goriely, Integrability and Nonintegrability of Dynamical Systems (World Scientific, Singapore, 2001)

32. R. Remmert, Theory of Complex Functions (Springer, New York, 1991)

33. B. O'Neill, Semi-Riemannian Geometry (Academic Press, New York, 1983)

34. R. Penrose, in Theoretical Principles in Astrophysics and Relativity, ed. by N.R. Lebovitz, W.H. Reid, P.O. Vandervoort (University of Chicago Press, 1978), pp. 217-43; also in General Relativity: An Einstein Centenary Survey, ed. by S.W. Hawking, W. Israel (CUP, 1979), chapter 12

35. V.A. Belinsky, I.M. Khalatnikov, E.M. Lifshitz, Adv. Phys. 31, 639-667 (1982)

36. S.W. Hawking, Commun. Math. Phys. 41, 199 (1975)

37. S.W. Hawking, Phys. Rev. D 14, 2460 (1976)

38. J.D. Bekenstein, Phys. Rev. D 7, 2333 (1973)

39. R. Penrose, Cycles of Time (The Bodley Head, London, 2010) 